\title{
Selfish Scheduler for Packet Scheduling Based on Packet Weighted Energy Drain Rate in Manets
}

\author{
${ }^{1}$ S. Lakshmi and ${ }^{2}$ S. Radha \\ ${ }^{1}$ Department of ECE, Sathyabama University, Chennai, India \\ ${ }^{2}$ Department of ECE, SSN College of Engineering, Chennai, India
}

Received 2012-10-10, Revised 2012-12-07; Accepted 2013-02-06

\begin{abstract}
The aim of our selfish scheduler based on packet weighted energy drain rate in Mobile ad-hoc networks mainly depends on the available least highest-limit of the residual value of a mobile node. The projected work comprises of mechanisms for detecting the selfish node as well as an attitude called rehabilitatebefore-malicious behavior for justifying against the malicious behavior of nodes having selfishness. It provides an immediate solution for separating the selfish nodes based on the parameter namely the packet weighted energy drain rate. If PWEDRSS is deployed in an ad hoc environment it not only provides an energetic and immediate solution to become aware of malicious nodes but also can detect selfish nodes which may be fruitless due the deficient energy available, since the restricted battery power is one of the main constraint for all the mobile nodes. The effective and well-organized performances of the projected work are done through ns-2 simulations. The parameters used are the Packet delivery ratio, Throughput, Control overhead, Total overhead and End to end delay. These parameters are computed based upon varying the number of nodes. The outcome forecast that the planned mechanism performs well.
\end{abstract}

Keywords: Ad Hoc Networks, Selfishness, Reliability, Energy Drain Rate

\section{INTRODUCTION}

Mobile ad hoc networks avails a collective and limited wireless medium which is employed by all the wireless nodes in the network providing efficient and effective control over this wireless medium is always significant. Several resource allocation attitudes have been contributed for achieving this task (Perkins et al., 2002). Although there are several such a resource scheduling algorithm, one of the packet scheduling strategy which considers the bandwidth for packet relay through multiple lines are projected (Broch et al., 1998). The above stated solutions primarily targets on get rid of the problems that could occur with multiple sessions while sharing a common wireless links (Das et al., 2000). Our Planned mechanism takes into account about the subjects that are distinct to MANET's namely dynamic topology and multihop environment
(Anuradha et al., 2009). The word "Packet Weighted" in our scheduler scheme infer the suggestion about the consumption of energy, which is directly proportional to the number of packets that are onward by an individual node. The scheduler considered is mostly used in the context of the selfish nodes, which refers to a mobile node that drops the packet that approach from other mobile nodes that drops the packets of its own node (Papadimitratos and Haas, 2002; Ghorpade et al., 2008). When we consider the routing of packet through Ad hoc On-Demand Distance Vector (AODV) routing protocol, the selfish node could show any one of the subsequent possible actions in Ad hoc network (Chen and Nahrstedt, 2004).

The consistency of the MANETs communication generally depends upon the implied trust between the nodes clearly trust in the sense refers to the full support which is create between nodes of the network so as to

Corresponding Author: S. Lakshmi, Department of ECE, Sathyabama University, Chennai, India 
ensure correct routing establishment techniques, through which the construction of routing information could be provided (Papadimitratos and Haas, 2002). But this kind of trust may be demoralized by adversary nodes that carry out security violated through attack nodes thus securing MANETs against MAC layer misbehavior has to be dealt and restrained.

\section{MATERIALS AND METHODS}

\subsection{Packet Weighted Energy Drain Rate Based Selfish Scheduler}

In our projected work, the main focus is on the reactive routing based protocols in which the route discovery is executed only when a node wants to establish a connection to transmit a data to another node. The routing protocol used for our study is the AODV, an on demand routing protocol.

If the source needs to communicate to a destination node, it generates data packets and transmits them through the Network layer, if there is no possible route from the source to the destination and then it initiates a route discovery process. We take into consideration that communication time is known. A node accepts to communicate the packet as shown in Fig. 1 only if it has at least Maximum-limit of Residual value.

\section{Algorithm 1: Choosing an Intermediate Node as Selfish Node:}

1. A Neighbor Node M receives a RREQ from source;

2. If session_life_time is implied in RREQ then

3. Check

4. If $\mathrm{NE}_{\mathrm{i}}>(\mathrm{PSWEDR} *$ session_life_time) then

5. Update life-duration field of $\bar{R}$ REQ.

6. Enable the communication and RREQ route to the Neighbor

7. Else

8. Disable the communication and drop the RREQs.

9. End if

10. Else

11. If $\mathrm{NE}_{\mathrm{i}}>\mathrm{MAX}$-LIMIT-RESIDUAL_VALUE then

12. Trigger the $M / \mathrm{m} / \mathrm{n}$ queue to schedule the packets.

13. Else

14. Presence of selfish node.

15. Trigger the $\mathrm{M} / \mathrm{m} / 1$ queue to schedule the packets.

16. End if

17. End if

\subsection{Packet Weighted Energy Drain Rate Computation}

Packet Weighted Energy Drain Rate (PWEDR) is calculated as the difference between the product of number of packets and the energy $E_{a}$ of the node and the product of number of packets and the energy $E_{b}$ of the node between a session divided by time interval Equation 1:

$\operatorname{PWEDR}\left(\mathrm{E}_{\mathrm{a}-\mathrm{b}}\right)=\frac{\mathrm{P}_{\mathrm{a}} * \operatorname{Engy}_{\mathrm{a}}-\mathrm{P}_{\mathrm{b}} * \operatorname{Engy}_{\mathrm{b}}}{\mathrm{t}_{\mathrm{b}}-\mathrm{t}_{\mathrm{a}}}$

When Engy $y_{a}$ and Engy $y_{b}$ are namely the nodes energy at $t_{a}$ and $t_{b}$ respectively. The PWEDR is averaged based on exponential averaging with $\alpha=0.75$.

To manipulate the Average Packet Weighted Energy Drain Rate (APWEDR) we use the formula Equation 2:

$$
\begin{aligned}
& \operatorname{APWEDR}\}=\alpha * \operatorname{PWEDR}(\mathrm{t})+(1-\alpha) \\
& * \operatorname{PWEDR}(\mathrm{t}-1)\left(\mathrm{E}_{\mathrm{a}-\mathrm{b}}\right)
\end{aligned}
$$

At the end, when the RREQ packet reaches the destination, it chooses a route that would maximize the life-time of the route by opting the one with maximum life-time of the node.

\section{Algorithm 2: Topology Maintenance by Rehabilitate-Before-Malicious Behaviour at Node N:}

1. Compute PWEDR periodically and check $\mathrm{NE}_{\mathrm{i}}$

2. If $\mathrm{NE}_{\mathrm{i}}<\mathrm{MIN}$-LIMIT-RESIDUAL_VALUE then

3. Check

4. If $\mathrm{N}==\mathrm{Non}-\mathrm{Selfish}$ node

5. Then

Relay the data packets to all the sources through node $\mathrm{N}$

7. End if

8. If $\mathrm{N}==$ Destination

9. Terminate the data replay by sending OCR to all sources that they are be in contact with the destination

10. End if

11. End if

The second Algorithm helps the protocol to route the packet and to rehabilitate quickly to link breakage due to presence of non-co-operating nodes when the energy of a node is fully drained i.e., when the current energy of the node goes below a minimum limit of residual value. The routing protocol reacts to this event and its feature depends in whether the node is a selfish node or a Destination Node. If the node is a selfish node, it sends Optimal Change Request (OCR) packets to all the source nodes through the neighbor's hops towards their respective destinations. 


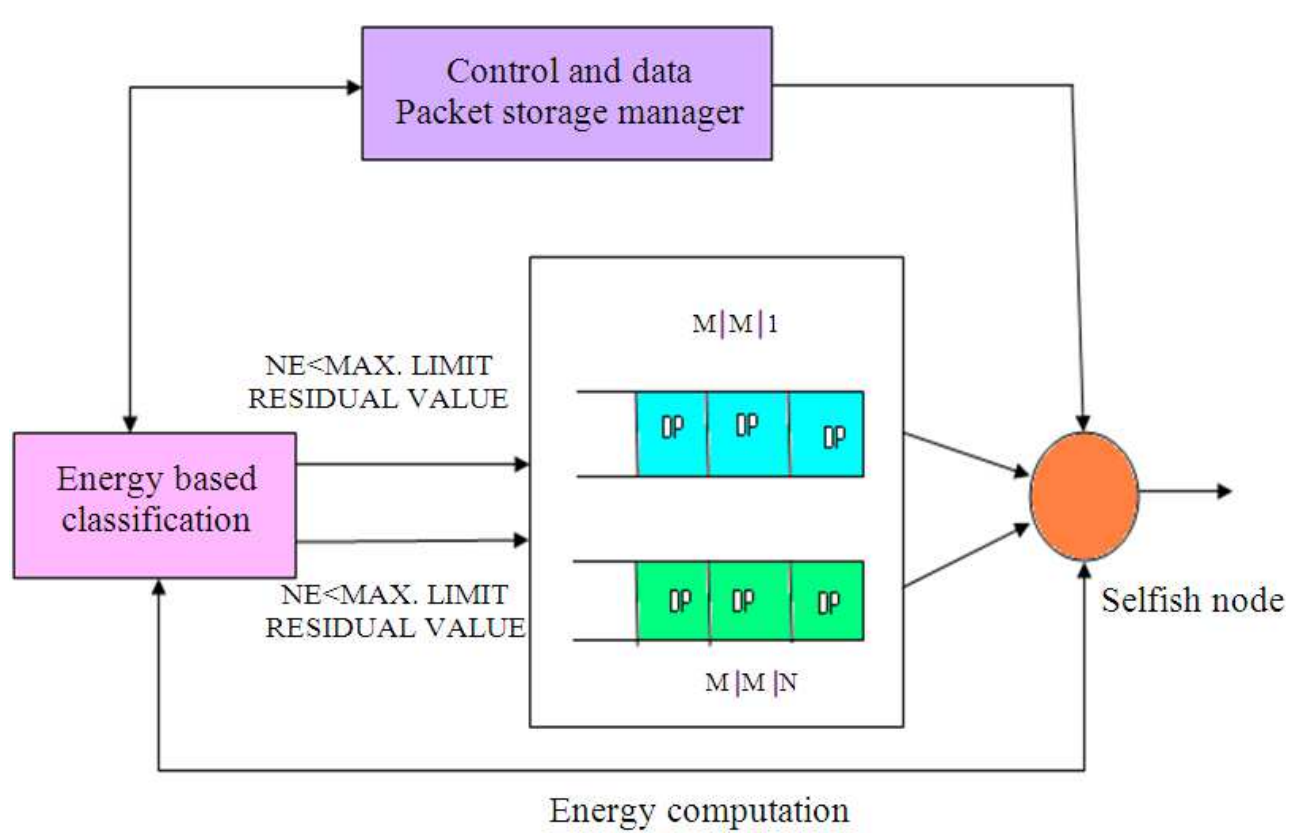

Fig. 1. Selfish sheduler scheme based on packet weighted energy drain rate

Table 1. Simulation parameters

\begin{tabular}{lll}
\hline Parameter & Value & Description \\
\hline Number of mobile nodes in the terrain & 50 Nodes & 50 nodes are placed in the network \\
Channel type required & Wireless ad-hoc channel type & Channel type \\
Propagation type & Two Ray ground model & The radio propagation model \\
Network interface type & Phy/WirelessPhy type & Network interface type \\
Interface queue type & $\mathrm{M} / \mathrm{M} / 1$ and $\mathrm{M} / \mathrm{M} / \mathrm{n}$ & Interface queue model \\
Antenna type & Omni-directional antenna & Antenna model \\
Protocol type & AODV & Ad-hoc on demand distance vector \\
Simulation time & $50 \mathrm{secs}$ & Maximum simulation time \\
Size of the packet & $512 \mathrm{bytes}$ & Variable data packet size \\
Dimensions of the terrain & $1000 \mathrm{~m} \times 1000 \mathrm{~m}$ & X and Y dimensions of the motion \\
\hline
\end{tabular}

The source node on receiving the OCR packet, Originates a new route discovery process for the communication session and hence with high chance obtains a fresh route before an actual link break occurs on the established original route. This could make the packet drops and increase the delay in time and hence enables the protocol to enhance rapidly to the network topology changes, if an alternate route to the desired destination exists. If the node being drained is a destination node, then it sends a request the source to stop all data routing to itself. When the request reaches the source, further replay of the data are prohibited. This may reduce the number of packet to be lost in the network and thus reduces packet delivery ratio and reduces resource usage by avoiding packet transmissions to unavailable destinations.

\section{RESULTS}

The Network Simulator intialisation is specified in Table 1. used for our study is ns-2.in our simulation 50 mobile nodes were placed randomly in a terrain area $1000 \times 1000 \mathrm{~m} .2 \mathrm{Mb} / \mathrm{s}$ is the wireless channel capacity used in our simulations. Each of the simulations is run for $50 \mathrm{sec}$.

The Packet Weighted based Energy drain rate scheduling scheme are applied by varying the number of mobile nodes and the packet size. The proposed methodology is compared with the selfish aware queue scheduler. For each and every node a Constant Bit Rate (CBR) source has been utilized. A node transmits varying number of packet size. The random way model is used for simulation. The maximum allowed speed for 
a node is $10 \mathrm{~m} \mathrm{sec}^{-1}$. The following performance metrics are used to compare the two scheduling algorithm are the packet delivery ratio, control overhead, total overhead, End-End delay and through put.

\subsection{Performance Metrics}

For performance estimation of this scheme the succeeding metrics are used.

\subsection{Packet Delivery Ratio}

Packet delivery ratio is the relation of the total number of data packets sent to the receivers to the total number of data packets received by the receivers.

\subsection{Control Overhead}

Control overhead is the relation of the total number of the control packets transmitted by the sender to the number of data packets delivered to the receivers.

\subsection{Total Overhead}

Total overhead can be defined as the total number of data packets and control packets transmitted to the total number of the data packets delivered.

\subsection{Throughput}

It may be defined as one of the dimensional parameter which represents the fraction of channel capacity utilized for reliable transfer of data from the source node to the destination nodes.

\subsection{End to End delay}

It may be defined as the time gap between the times of packet origin to the time upto the last bit arrival of the packet to the target.

\subsection{Performance Evaluation of SSQM 3.7.1. Packet Delivery Ratio}

Figure 2 shows the comparison chart between the Packet delivery ratio and the number of mobile nodes for two strategies, they are without PWEDRSS and with PWEDRSS. The graphical illustration apparently describes that the decrease in packet delivery ratio is due the increase in selfish behavior. When the reactive scheduling mechanism is deployed packet delivery ratio increases.

The chart make known that the packet delivery ratio get decreased in the network when the selfish node behavior takes place but can be increased on an average by $38 \%$ when the PWEDRSS strategy is provided to isolating selfish node using weighted energy drain rate for the packet. The packet delivery ratio obtained through this solution is optimal and efficient.

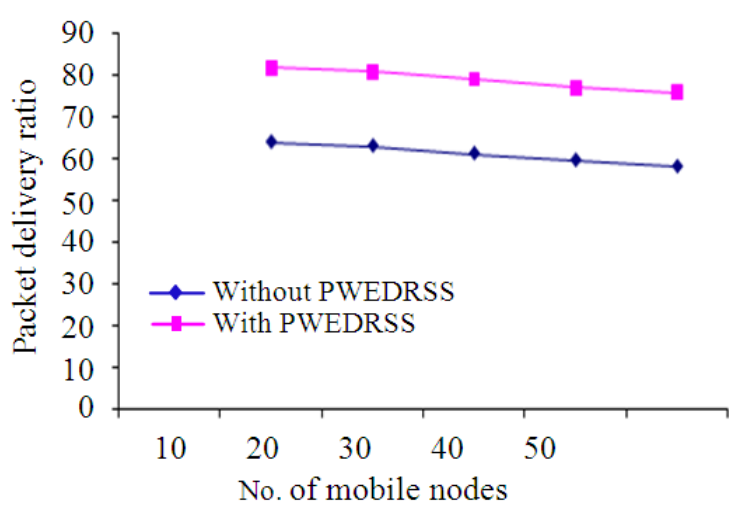

Fig. 2. Packet delivery ratio for PWEDRSS

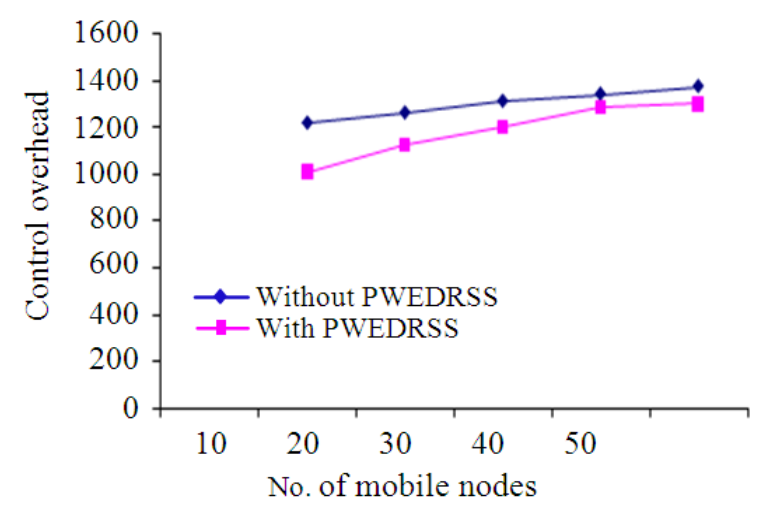

Fig. 3. Control overhead for PWEDRSS

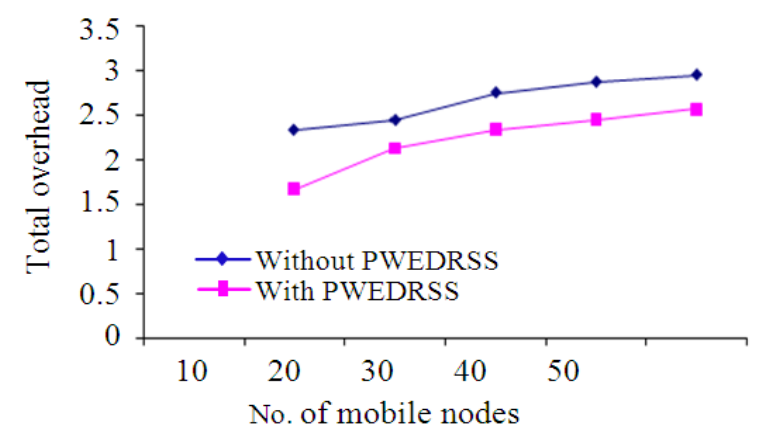

Fig. 4. Total overhead for PWEDRSS

\subsection{Control Overhead}

Figure 3 depicts the shows the comparison chart between the control overhead and the number of selfish nodes for two strategies, they are without PWEDRSS and with PWEDRSS. The graphical illustration apparently describes that the increase in control overhead, with the increase in selfish behavior. When the reactive scheduling mechanism is deployed control overhead decreases. 


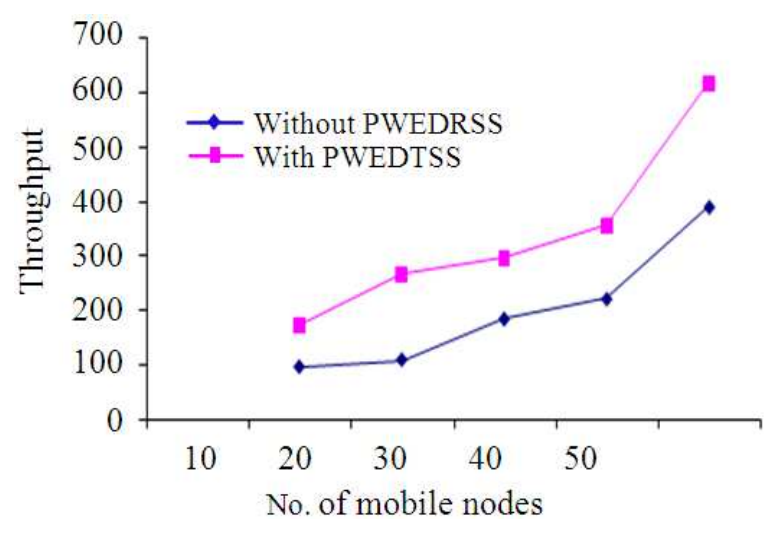

Fig. 5. Throughput for PWEDRSS

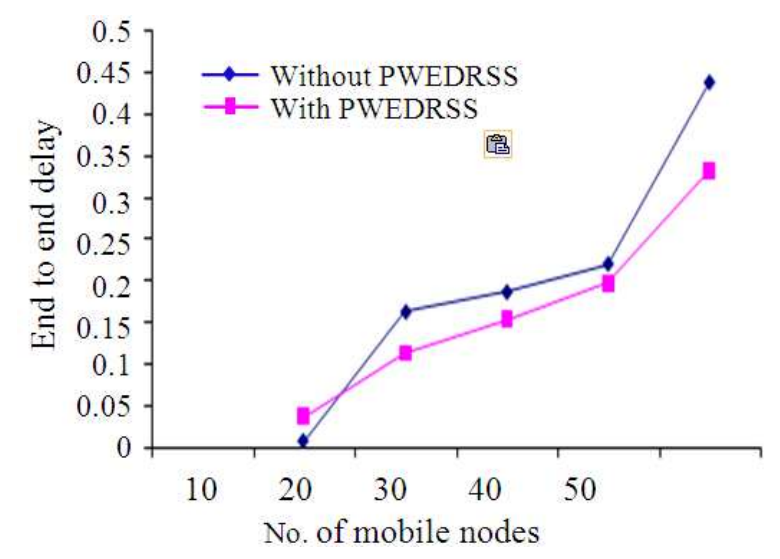

Fig. 6. End to end delay for PWEDRSS

The chart reveals that the control overhead ratio get decreased in the network when the selfish node behavior takes place but can be increased on an average by $17 \%$ when the PWEDRSS strategy is provided to isolating selfish node using weighted energy drain rate for the packet.

\subsection{Total Overhead}

Figure 4 shows the comparison chart between the Total overhead and the number of selfish nodes for two strategies, they are without PWEDRSS and with PWEDRSS. The graphical illustration apparently describes that the increase in Total overhead due to the increase in selfish behavior. When the reactive scheduling mechanism is deployed total overhead decreases.

The graph portrays that the Total overhead get increased in the network when the selfish node behavior takes place but can be decreased on an average by $21 \%$ when the PWEDRSS strategy is provided to prevent the attack.

\subsection{Throughput}

Figure 5 shows the comparison chart between the throughput and the number of selfish nodes for two strategies, they are without PWEDRSS and with PWEDRSS. The graphical illustration apparently describes that the decrease in throughput due to the increase in selfish behavior. When the reactive scheduling mechanism is deployed throughput increases.

The graph portrays that the throughput get decreased in the network when the selfish node behavior takes place but can be increased on an average by $22 \%$ when the PWEDRSS strategy is provided to prevent the attack.

\subsection{End to End delay}

Figure 6 shows the comparison chart between the End to End Delay and the number of selfish nodes for two strategies, they are without PWEDRSS and with PWEDRSS. The graphical illustration apparently describes that the increase in end to end delay due to the increase in selfish behavior. When the reactive scheduling mechanism is deployed end to end delay decreases.

The graph portrays that the end to end delay get increased in the network when the selfish node behavior takes place but can be decreased on an average by $28 \%$ when the PWEDRSS strategy is provided.

\section{DISCUSSION}

A new scheduling algorithm Packet weighted Energy Drain Rate based Selfish Scheduler (PWEDRSS) was discussed related to the parameters throughput,end to end delay,packetdelivery ratio,control overhead and total overhead by varying themobile nodes and it is proved that PWEDRSS performs better.

\section{CONCLUSION}

In this study, Packet weighted energy based scheduling strategy is revealed. This mechanism work out the energy based on the number of packets transmitted by a node, through which scheduling decision could be made. By means of simulation, the algorithmic performance can be compared. Evolution analysis foretells that this solution performs better. In the near future, this mechanism can be used to prevent traffic congestion. 


\section{REFERENCES}

Anuradha, S., G. Raghuram, K.E.S. Murthy, B.G. Reddy, 2009. New routing technique to improve transmission speed of data packets in point to point networks. ICGST-CNIR J.

Broch, J., D.A. Maltz, D.B. Johnson, Y.C. Hu and J. Jetcheva, 1998. A performance comparison of multi-hop wireless ad hoc network routing protocols. Proceedings of the 4th annual ACM/IEEE International Conference on Mobile Computing and Networking, Oct. 25-30, ACM Press, Dallas, TX, USA., pp: 85-97. DOI: $10.1145 / 288235.288256$

Chen, K. and K. Nahrstedt, 2004. iPass: An incentive compatible auction scheme to enable packet forwarding service in MANET. Proceedings of 24th International Conference on Distributed Computing System, Mar. 26-26, IEEE Xplore Press, pp: 534542. DOI: 10.1109/ICDCS.2004.1281620
Das, S.R., C.E. Perkins and E.M. Royer, 2000. Performance comparison of two on-demand routing protocols for ad hoc networks. Proceedings of the 19th Annual Joint Conference on IEEE Computer and Communications Societies, Mar. 26-30, IEEE Xplore Press, Tel Aviv, pp: 3-12. DOI: 10.1109/INFCOM.2000.832168

Ghorpade, V.R., Y.V. Joshiand and R.R. Manthalkar, 2008. Fuzzy logic based trust management framework for MANET. DSP J.

Papadimitratos, P. and Z.J. Haas, 2002. Secure routing for mobile ad hoc networks. Proceedings of the SCS Commnication Networks and Distributed Systems Modeling and Simulation Conference (CNDS), Jan. 27-31, San Antonio, TX., pp: 193-204.

Perkins, C., E.B. Royer and S. Das, 2002. Ad hoc OnDemand Distance Vector (AODV) routing. University of Cincinnati. 